\title{
AUTOMATIC VIDEO DETECTION OF NOCTURNAL EPILEPTIC MOVEMENT BASED ON MOTION TRACKS
}

\author{
Kris Cuppens ${ }^{1,2}$, Bert Bonroy ${ }^{1}$, Anouk Van de Vel $^{3}$, Berten Ceulemans ${ }^{3}$, Lieven Lagae ${ }^{4}$, Tinne \\ Tuytelaars ${ }^{5}$, Sabine Van Huffel ${ }^{2,6}$, Bart Vanrumste ${ }^{1,2}$ \\ ${ }^{I}$ Mobilab, K.H.Kempen, Geel, Belgium \\ ${ }^{2}$ KULeuven, ESAT, SISTA, BioMed, Leuven, Belgium \\ ${ }^{3}$ UZAntwerpen, Child neurology, Edegem, Belgium \\ ${ }^{4}$ UZLeuven, Pediatrics, Leuven, Belgium \\ ${ }^{5}$ KULeuven, ESAT, PSI, Leuven, Belgium \\ ${ }^{6}$ KU Leuven-IBBT Future Health Department, Belgium \\ kris.cuppens@khk.be
}

\begin{abstract}
Keywords: epilepsy, seizure detection, image motion analysis, video monitoring, optical flow, mean shift clustering
Abstract: Epileptic seizure detection in a home situation is often not feasible due to the complicated attachment of the EEG-electrodes on the scalp. We propose to detect nocturnal seizures with a motor component in patients by means of a single video camera. To this end we use a combination of optical flow and mean shift clustering to register moving body parts. After extraction of seven features, related to amplitude, duration and direction of the motion, we carry out a first validation with a linear support vector machine classifier. This resulted in a sensitivity of $80.60 \%$ and a positive predictive value of $62.07 \%$.
\end{abstract}

\section{INTRODUCTION}

Epilepsy is one of the most common neurological disorders in the world, that affects approximately one percent of the world's population. In $80 \%$ of the patients the seizures can be controlled by medication or surgery. In the other group (20\%), patients need to be monitored on a regular basis to follow up their disease, especially during the night when there is no social control by the patient's environment. However knowing the number of seizures during the night would provide the neurologist with an objective measure to alter the medication and increase the quality of life of the patient. A second advantage of a nocturnal monitoring system is that an alarm can be given if a heavy seizure occurs which needs care afterwards.

Currently the most widely used method for monitoring epilepsy is video/EEG-monitoring. But the attachment of EEG-electrodes, which measure the electrical activity of the brain, is complicated, labor intensive and mostly restricted to a clinical setting. For monitoring patients in a home setting, a more practical setup is required.
Monitoring a patient with a video recording system can offer a solution, as it monitors the patient without making physical contact and it is easy to install in the bedroom.

Human motion analysis has already been studied extensively in other applications such as gesture recognition or surveillance (Hu et al, 2004.)( Turaga et al., 2008). The nocturnal detection of seizures is in some points more challenging. We cannot use the skin color information, which is often used in gesture analysis, due to the gray scale images because of night vision. Also, detecting the patient's extremities is most of the time not feasible because the patients are covered with a blanket. And finally, there are different types of motor manifestations in seizures. Therefore we focus on one type of seizure only, namely the myoclonic seizure. Even then, there is a considerable variation in manifestation within a dataset of one patient and the datasets between patients.

In Cuppens et al. (Cuppens et al., 2010) body movement is detected in nocturnal recordings from epileptic patients as a data reduction step. The optical flow based method reaches a sensitivity of 
$100 \%$ and a positive predictive value between $82 \%$ and $100 \%$ when testing it on three test sets.

Karayiannis (Karayiannis et al., 2006) already studied the detection of seizures in neonates, with a focus on focal clonic and myoclonic seizures. The patients were monitored in the neonatal intensive care unit where all the body parts were clearly visible for the camera. The motion of the body parts was quantified by temporal motion-strength signals based on optical flow computation, and by motion trajectories. The classification based on neural networks resulted in a sensitivity and specificity above $90 \%$.

The difference in our study is that we focus on a different patient group. Furthermore, the setting is as natural as possible, i.e. the patients can be covered with a blanket and we do not make use of markers, just as in a normal home situation. And finally, we also make use of new features that we extract from the motion tracks.

\section{METHOD}

\subsection{Data acquisition}

The data was acquired at the Pulderbos rehabilitation centre for children and youth. In this centre, epileptic patients up to the age of 18 years are monitored. Video data is acquired during the night with a near-infrared camera. The frame rate is 25 frames per second and the resolution is 320 by 240 pixels. The datasets are labelled by an expert based on the video and EEG recordings.

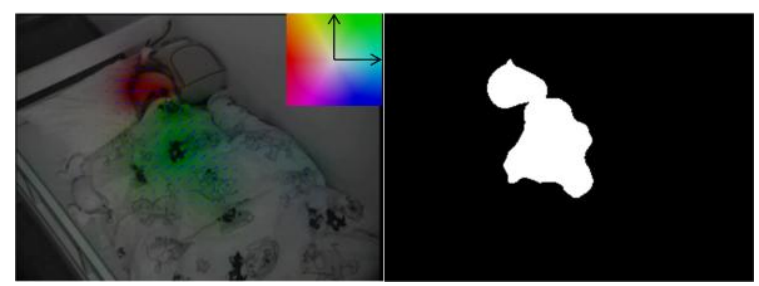

Figure 1: motion vector field in one frame from epileptic patient, the colors represent the direction of motion vectors (a) the frame after thresholding (b).

\subsection{Motion detection}

The extraction of motion from the video sequences is carried out by the Horn-Schunck optical flow calculation (Horn and Schunck, 1981). The motion vector field is thresholded to remove low amplitude noisy motion vectors and to get a first segmentation, as shown in figure 1 .

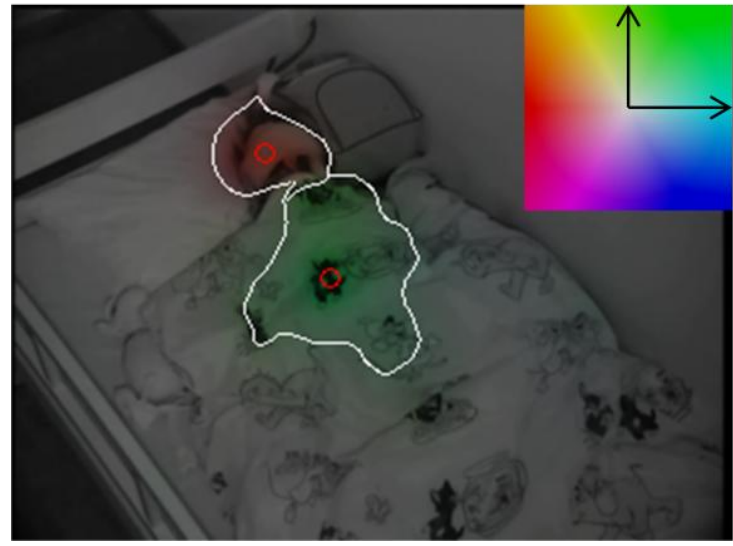

Figure 2: result after clustering the motion vectors. The red circles indicate the modes of the clusters. The colors indicate here the direction of the motion.

\subsection{Spatial clustering}

In a next step the motion vectors are clustered in homogenous blobs with similar motion directions. This is realized by a slightly modified version of the mean-shift clustering algorithm (Cheng, 1995) (Fukunaga and Hostetler, 1975), which was proposed in (Min et al., 2008) for the classification of movements in ballet sequences.

The mean shift algorithm is an iterative procedure that starts in a pixel in the segmente $d$ area. In the next iteration the point moves to a new location according to eqn. (1).

$$
m(p)=\frac{\sum_{q \in I} K(p-q) F(q) w(q) q}{\sum_{q \in I} K(p-q) F(q) w(q)},
$$

where $p$ is the original location, $q$ are the pixels in image $I, K$ is the chosen kernel, $F$ is the amplitude of the motion vector and $w$ is a weight function based on vector similarity.

The point moves towards the location with the local maximal vector amplitude (mode). All the points in the segmented frame that iterate to the same local maximum belong to the same cluster.

As a kernel we use a parabolic function with a fixed bandwidth. The weight function $w$ gives a measure for the similarity of the vector directions, with a value of zero if the angle between the two vectors is $180^{\circ}$ and one if the angle is $0^{\circ}$. This way, vectors with a different direction are not clustered together.

Figure 2 shows the result of clustering the frame from figure 1. The two parts in the frame with a different movement direction are well split up. 


\subsection{Tracking}

After the motion vectors in the frames are clustered, they are tracked over time based on the location of the cluster modes, as explained in (Crocker and Grier, 1996), assuming that the location of the object would not change too much from one frame to another (proximity) and the maximum velocity defines an upper bound on the object velocity and limits the possible correspondences to a circular neighborhood around the object (Yilmaz et al., 2006). If the tracked body part stops moving for more than half a second, the movement is split up in two tracks, otherwise they are considered as one.
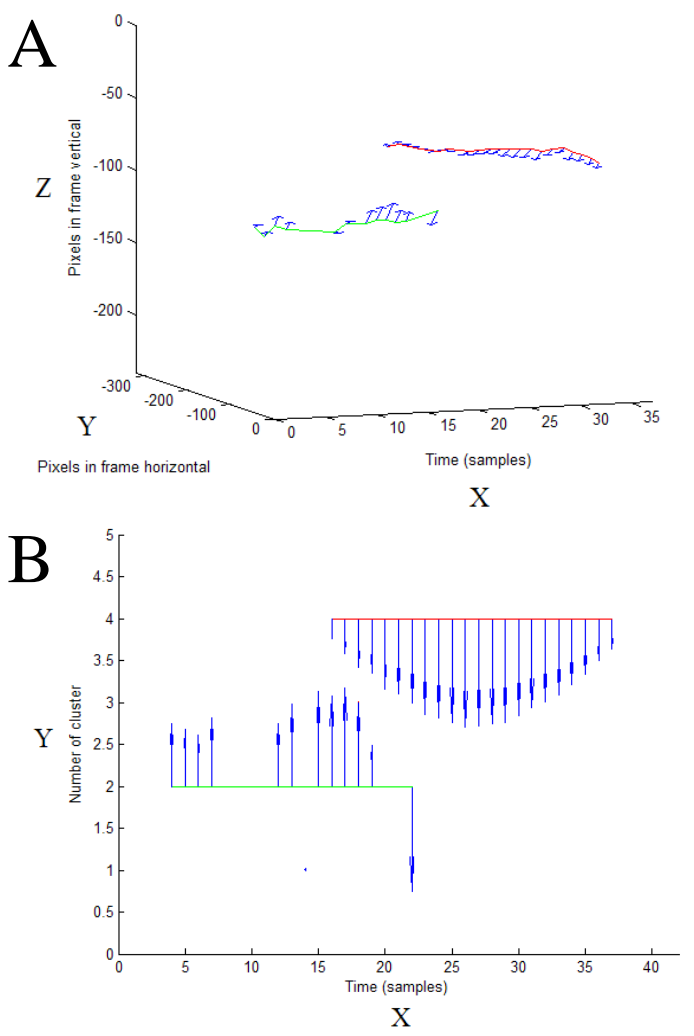

Figure 3: motion tracks (green and red) with the corresponding motion vectors in blue, the YZ-plane represent the locations in the image frame, the $\mathrm{X}$-direction corresponds to the time (a) motion tracks represented with the amplitude and sign ( $\mathrm{Y}$-axis) and time (X-axis) (b).

\subsection{Feature extraction}

From every cluster in every frame the average motion vector is calculated. Every motion track results in a series of two dimensional motion vectors from which we derive different features. This is visualized in figure 3 .

Because the myoclonic shocks are characterized by a short and intense movement of the limbs, mostly in arms or shoulders, in one direction followed by a movement in the opposite direction, e.g. the relaxation after the contraction of the arms muscles, we incorporate features that quantify the change in motion direction.

Therefore we use two features covering the change in direction, namely slow and fast phase changes. Other features we use are the duration of the movement, the maximal, median and average vector amplitude and the number of vectors in the track. The number of vectors is sometimes smaller than the length because the body part can stop moving for up to half a second and still be in the same track.

\subsection{Classification}

The classification of the seizures is carried out by a support vector machine (SVM) classifier. We use a linear kernel on all seven features for classification. This classification was done on one training and test set, to get our first preliminary results. Notice that the classification was performed on the tracks, not on the seizures as a whole.

\section{RESULTS}

Table 1: feature values of tracks.

\begin{tabular}{|c|c|c|c|c|c|c|c|}
\hline & 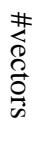 & 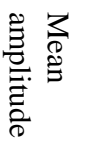 & 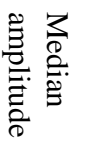 & 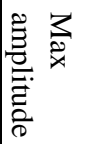 & 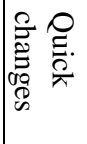 & 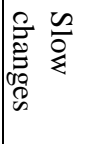 & 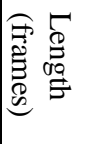 \\
\hline \multicolumn{8}{|c|}{ Normal movement } \\
\hline$\# 1$ & 3 & 0.67 & 0.71 & 0.73 & 0 & 0 & 3 \\
\hline \#2 & 48 & 0.72 & 0.75 & 0.95 & 0 & 2 & 49 \\
\hline \#3 & 15 & 0.43 & 0.47 & 0.52 & 2 & 0 & 33 \\
\hline \#4 & 6 & 0.71 & 0.71 & 0.84 & 0 & 0 & 7 \\
\hline \#5 & 6 & 0.41 & 0.39 & 0.48 & 0 & 0 & 6 \\
\hline \multicolumn{8}{|c|}{ Epileptic movement } \\
\hline$\# 1$ & 6 & 0.26 & 0.28 & 0.37 & 1 & 0 & 8 \\
\hline$\# 2$ & 5 & 0.09 & 0.10 & 0.11 & 1 & 0 & 7 \\
\hline \#3 & 33 & 0.20 & 0.13 & 0.59 & 0 & 1 & 44 \\
\hline \#4 & 4 & 0.38 & 0.42 & 0.51 & 2 & 0 & 7 \\
\hline \#5 & 12 & 0.11 & 0.12 & 0.22 & 0 & 0 & 24 \\
\hline
\end{tabular}

Some typical values for the track features can be found in table 1 . This table shows the values for 5 epileptic and 5 non-epileptic tracks from motions.

For the validation of our approach we use a training set containing 100 motion tracks from normal nocturnal movement from an epileptic patient, and 113 tracks from 10 myoclonic shocks. 
All the data is from one patient. During the time that a seizure occurs, all the tracks are labeled as epileptic. The test set consists also of 100 normal motion tracks and 67 epileptic motion tracks from 5 myoclonic seizures. For the time being, the classification is based on the single tracks, so not on the whole seizures, thus we have 113 positive samples in the training set and 67 in the test set, each of them being labeled as part of a myoclonic shock.

After training the linear SVM model once, we tested it on our test set which resulted in 54 true positives, 67 true negatives, 33 false positives and 13 false negatives. This corresponds to a sensitivity of $80.60 \%$, a Positive Predictive Value of $62.07 \%$ and a specificity of $67.00 \%$.

\section{DISCUSSION}

The classification is carried out on individual motion tracks. The motion tracks extracted during an epileptic shock are possibly not all from the epileptic movement itself, but also from e.g. movements of the bed because of the seizure. So the classification can be improved on this point. Moreover, the features from different tracks originating from one seizure can be combined, to further improve the detection.

The training and testing is now performed on a small dataset. To have more solid validation, a larger dataset should be used. These results are preliminary but give an indication that the detection of specific types of seizures by the proposed algorithm is possible.

The obtained results in this paper are less optimal than in (Karayiannis et al., 2006), namely a sensitivity of $80.60 \%$ and a specificity of $67.00 \%$ compared to a sensitivity and specificity above $90 \%$ in (Karayiannis et al., 2006). But the circumstances in our setup were more difficult as the patients' body parts are most of the time not clearly visible. Removing the blankets is not an option as it would reduce the sleeping quality of the patients too much. But notice that there is still some room for improvement in our algorithm.

\section{CONCLUSIONS}

The detection of seizures based on motion tracks extracted from the optical flow calculation and the mean shift clustering algorithm is possible. In the first test on 15 myoclonic shocks a sensitivity of $80.60 \%$ and a positive predictive value of $62.07 \%$ is reached. Further research is required to confirm these first results and to test the algorithm on other seizures.

\section{ACKNOWLEDGEMENTS}

Research supported by Research Council KUL: GOA-MANET, IWT: TBM070713-Accelero, Belgian Federal Science Policy Office IUAP P6/04 (DYSCO, 'Dynamical systems, control and optimization, 2007-2011); EU: Neuromath (COSTBM0601). Kris Cuppens is funded by a Ph.D grant of the Institute for the Promotion of Innovation through Science and Technology in Flanders (IWTVlaanderen)

\section{REFERENCES}

Karayiannis N.B., Xiong Y., Tao G., Frost J.D. Jr., Wise M.S., Hrachovy R.A. and Mizrahi E.M., Automated detection of videotaped neonatal seizures of epileptic origin. Epilepsia, vol. 47, pp. 966-980, 2006.

Min J. H., Kasturi R. and Camps O., Extraction and temporal segmentation of multiple motion trajectories in human motion. Image and Vision Computing, vol. 26, pp. 1621-1635, 2008

Crocker J. C. and Grier D. G., Methods of digital video microscopy for colloidal studies. Journal of Colloid and Interface Science, vol. 179, pp. 298-310, 1996.

Yilmaz A., Javed O. and Shah M., Object tracking: A survey. Acm Computing Surveys, vol. 38, 2006.

Horn B. K. P. and Schunck B. G., Determining OpticalFlow. Artificial Intelligence, vol. 17, pp. 185-203, 1981.

Cuppens K., Lagae L., Ceulemans B., Van Huffel S. and Vanrumste B., Automatic video detection of body movement during sleep based on optical flow in pediatric patients with epilepsy. Medical \& biological engineering \& computing, vol. $48, \mathrm{~N}^{\circ}$ 9, pp. 923-931, 2010.

Cheng Y. Z., Mean Shift, Mode Seeking, And Clustering. IEEE Transactions on Pattern Analysis and Machine Intelligence, vol. 17, pp. 790-799, 1995.

Fukunaga K. and Hostetler L. D., Estimation Of Gradient Of A Density-Function, With Applications In PatternRecognotion. IEEE Transactions on Information Theory, vol. 21, pp. 32-40, 1975.

Hu W. M., Tan T.N., Wang L., Maybank S., A survey on visual surveillance of object motion and behaviors. IEEE Transactions on Systems Man and Cybernetics Part C-Applications and Reviews, vol. 34, pp. 334352, 2004

Turaga P., Chellappa R., Subrahmanian V.S., Udrea O., Machine Recognition of Human Activities: A Survey. IEEE Transactions on Circuits and Systems for Video Technology, vol. 18, pp. 1473-1488, 2008. 